\title{
Catéteres con resenvorio para uso prolongado
}

González-Fisher RF, ${ }^{*}$ Montiel-Mendoza ZJ **, Marín-Magallanes C ***

\begin{tabular}{|c|c|}
\hline Resumen & Abstract \\
\hline $\begin{array}{l}\text { La instalación de catéteres de silicón con reservorio } \\
\text { subcutáneo para uso prolongado han mejorado sustan- } \\
\text { cialmente la calidad de vida de los pacientes sometidos } \\
\text { a quimioterapia antineoplásica, tratamiento antibiótico } \\
\text { prolongado, nutrición parenteral total o aquellos que } \\
\text { requieren de tomas frecuentes de sangre para estudios } \\
\text { de laboratorio, sin embargo, su uso no está libre de } \\
\text { complicaciones, presentamos la experiencia de un gru- } \\
\text { po quirúrgico con } 100 \text { catéteres en } 98 \text { pacientes con } \\
\text { seguimiento mínimo de } 6 \text { meses, y discutimos las com- } \\
\text { plicaciones encontradas y posibles formas de evitarlas. } \\
\text { LUXMÉDICA 7(20): LUXMÉDICA 7(20): } 21 \text {-27 }\end{array}$ & $\begin{array}{l}\text { The use of silicon catheters with a subcutaneous im- } \\
\text { platable device for long periods of time have made } \\
\text { substantial improvements on quality of life on patients } \\
\text { who require chemotherapy, long term antibiotics, total } \\
\text { parenteral nutrition or frequent venous punctures to } \\
\text { obtain blood for laboratory analysis, despite this, the } \\
\text { use of these devices is not free of complications, we } \\
\text { present the experience of a single surgical group with } \\
\text { XXX catheters on XX patients with a followup of at } \\
\text { least } 6 \text { months, and we discuss complications found } \\
\text { and ways to prevent them. LUXMÉDICA 7(20): LUXMÉ- } \\
\text { DICA 7(20): } 21-27\end{array}$ \\
\hline $\begin{array}{r}\text { Palabras Clave: Port-a cath, catéter, com- } \\
\text { plicaciones }\end{array}$ & $\begin{array}{l}\text { Key Words: Port-a-cath, catéter, } \\
\text { complications }\end{array}$ \\
\hline
\end{tabular}

\section{Introducción}

Un acceso venoso confiable es necesario para pacientes que requieren de quimioterapia antineoplásica, antibióticos por períodos prolongados, nutrición parenteral total o venopunciones frecuentes con fines diagnósticos. ${ }^{1,2}$

El uso de accesos vasculares por períodos prolongados se ha incrementado y sobre todo ha mejorado la calidad de vida de los pacientes con cáncer ${ }^{3}$, el advenimiento de catéteres de silicón co-

* Cirujano General / Cirujano Oncólogo, Central Médico Quirúrgica de Aguascalientes

** Médico Interno de Pregrado, Central Médico Quirúrgica de Aguascalientes

*** Cirujano General. Central Médico Quirúrgica de Aguascalientes

Fecha de recibido: 21 de febrero 2012

Fecha de aceptación: 12 de marzo 2012

Correspondencia Dr. Ricardo F. González Fisher FACS (MAC) Central Médico Quirúrgica de Aguascalientes, República de Perú \# 102, Las Américas 20230, Aguascalientes, México. Correo electrónico cancer@aguascalientes.com 
locados mediante un túnel subcutáneo desarrollados por Broviac y Hickman fue seguido por puertos que se colocan por debajo de la piel que han permitido la medicación cómoda y segura..$^{3,4}$ Sin embargo, a pesar de estos avances se siguen presentando complicaciones que en ocasiones pueden poner en peligro la vida de los pacientes o retrasar los tratamientos. ${ }^{5,6}$

El propósito de este estudio es analizar la experiencia personal con 110 catéteres con seguimiento mínimo de un año, evaluar las complicaciones y opciones para evitarlas.

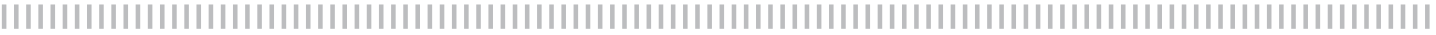

\section{Material y métodos}

Se hizo un estudio retrospectivo de los pacientes en quienes se colocó un catéter con reservorio subcutáneo para uso prolongado registrados en las libretas de programación de cirugía en el Hospital Central Médico Quirúrgica de Aguascalientes entre

junio de 1999 y junio de 2011, se obtuvo información en el expediente clínico del hospital y consulta externa, para hacer un estudio descriptivo incluyendo datos demográficos, diagnóstico de ingreso, vía de acceso, seguimiento y complicaciones.

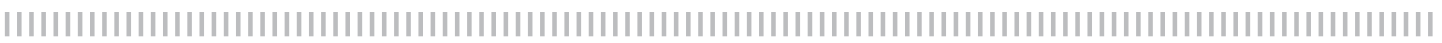

\section{Resultados}

Se revisó el expediente de 93 pacientes en quienes se instalaron 99 catéteres. La población estudiada constó de 70 mujeres y 23 hombres con un rango de edad entre
12 y 81 años de edad, con una media de 56.47 y una mediana de 60 años.

Los diagnósticos de ingreso se presentan en la tabla 1.

\section{Tabla |}

Diagnóstico de Ingreso en 93 pacientes que requirieron colocación de catéter con reservorio para uso prolongado

\begin{tabular}{|l|l|}
\hline Cáncer de Mama & 46 \\
\hline Cáncer de Pulmon & 8 \\
\hline Cáncer de Ovario & 7 \\
\hline Cáncer de Colón & 6 \\
\hline Cáncer Hematológico & 4 \\
\hline Cáncer de Páncreas & 3 \\
\hline Primario desconocido & 3 \\
\hline Cáncer de Próstata & 2 \\
\hline Cáncer Testicular & 2 \\
\hline Cáncer Cutáneo No Melanoma & 2 \\
\hline Cáncer de Esófago & 2 \\
\hline Cáncer Gástrico & 2 \\
\hline Feocromocitoma Metastásico & 1 \\
\hline Hepatocarcinoma & 1 \\
\hline Melanoma & 1 \\
\hline Cáncer Cervicouterino & 1 \\
\hline Sarcoma de Partes Blandas & 1 \\
\hline Carcinomatosis Peritoneal & 1 \\
\hline
\end{tabular}


90 catéteres fueron colocados por venodisección de la vena cefálica en el surco deltopectoral, 60 de ellos en el lado derecho y 30 en el izquierdo, en 7 pacientes fue necesario colocar el catéter por punción subclavia y en 2 pacientes se uso acceso por punción yugular. Todos los pacientes tuvieron seguimiento por el mismo grupo al menos por seis meses, el catéter se re- tiró por haber completado su vida útil en 70 pacientes, en seis pacientes aún está en uso, en dos pacientes se colocó un segundo catéter por recurrencia tumoral, en cuatro más por complicaciones del catéter (tabla 2) y 17 pacientes fallecieron con el catéter in situ.

Las complicaciones encontradas en 99 catéteres se presentan en la tabla 2 .

\section{Tabla 2}

Complicaciones en 99 catéteres con reservorio subcutáneo para uso prolongado

\begin{tabular}{|lcl|}
\hline Complicaciones mayores & Número de pacientes & Resolución \\
\hline Oclusión total & 1 & Trombolisis fallida, cambio de catéter \\
\hline Ruptura & 1 & Retiro y cambio de catéter \\
\hline Infección & 1 & Retiro de catéter, antibióticos, nuevo catéter \\
\hline Exposición del puerto & 1 & Cambio de catéter \\
\hline Expulsión de la vena & 2 & Retiro de catéter \\
\hline & & \\
Complicaciones menores & Número de pacientes & Resolución \\
\hline Oclusión parcial & 8 & Se continuó tratamiento \\
\hline Rotación del puerto & 2 & Recolocación del puerto \\
\hline
\end{tabular}

\section{Discusión}

El uso de catéteres permanentes con reservorio subcutáneo (Figura1) ha venido a revolucionar el manejo de los pacientes que requieren de tratamientos parenterales por períodos prolongados, particularmente para quimioterapia, nutrición parenteral y tomas frecuentes de sangre, la gran utilidad de estos catéteres ha atraído la atención de diversas especialidades como radiología intervencionista, ginecología, anestesiología y neurología entre otras ${ }^{3}$, esto ha condicionado que un procedimiento que se realizaba por cirujanos a través de disección de la vena cefálica en el surco deltopectoral, sea abordado con mayor frecuencia a través de técnicas percutáneas que no requieren de experiencia quirúrgica.?

Las técnicas percutáneas más utilizadas son la punción subclavia y la punción yugular, ambas tiene la ventaja de poder realizarse sin un entrenamiento quirúrgico, sin embargo tienden a tener mayor frecuencia de complicaciones como neumotórax, ruptura del catéter o bradicardia. Jablon y cols hicieron una revisión retrospectiva de los catéteres colocados en el Albert Einstein Medcial Center de Filadelfia entre 1988 y 2001 encontrando una frecuencia global de complicaciones del $14 \%$, siendo más frecuentes en los pacientes con técnica percutánea, por lo que concluyeron que la venodisección es más segura para el paciente y debería de considerarse como el método de elección. ${ }^{8}$ De igual forma un estudio prospectivo de Knebel y cols ${ }^{9}$, que incluyó a 110 pacientes encontró mayor frecuencia de complicaciones en la técnica percutánea aunque sin una diferencia significativa. Finalmente Di Carlo y cols, después de una extensa revisión de la literatura, concluyen que después de 27 años de experiencia con 11430 de estos implantes, la venodisección es el único método 
seguro para evitar complicaciones potencialmente letales. ${ }^{3}$ Sin embargo, Galloway y Bodenham - anestesiólogos- prefieren la inserción percutánea por tener "mejor resultado cosmético y menos infecciones locales", de igual manera consideran que a través de la vía quirúrgica se inutiliza definitivamente una vena, aunque la frecuencia de neumotórax cuando se coloca el catéter por punción percutánea es mayor. ${ }^{10}$

En nuestra experiencia la colocación del catéter fue exitosa por vía quirúrgica -venodisección- en 90 pacientes sin complicaciones perioperatorias; sin embargo, no fue posible canalizar la vena cefálica en nueve casos, debido a que tenían un calibre muy pequeño, o estaba sustituída por un plexo venoso, o dañada por quimioterapia previa, por lo que se optó por un acceso percutáneo a través de punción subclavia en siete pacientes y yugular en dos. Se ha descrito ausencia de la vena cefálica hasta en 3\% de los varones y $1 \%$ de las mujeres. ${ }^{11}$

Encontramos un caso de compresión y ruptura del catéter entre la primera costilla y la clavícula (Figura 2) en un paciente en quien se colocó el catéter por punción subclavia, esto ha sido publicado por otros. ${ }^{10,12,13}$ El pinzamiento del catéter "Pinch off" se refiere al atrapamiento de catéteres subclavios entre la primera costilla y la clavícula (Figura 3 ), con el paso del tiempo y después de compresión repetida se causa ruptura del catéter que ocasiona extravasación de líquidos -como en nuestro caso-, fractura y embolización de la parte intravascular del catéter; este fenómeno se presenta en 1 a $5 \%$ de los pacientes con catéteres colocados por vía percutánea a través de punción subclavia. ${ }^{10,12}$ Clínicamente esta complicación puede sospecharse cuando se encuentra dificultad para el retorno venoso o resistencia en la inyección de soluciones -con frecuencia relacionada con la posición del paciente- o sensación de malestar y aumento de volumen con la infusión de líquidos. Cuando se sospecha pinzamiento del catéter se debe de comprobar mediante radiografía de tórax, que mostrará el sitio de la compresión (figura 4). ${ }^{14}$ Se ha sugerido que este problema puede evitarse mediante punción más lateral de la subclavia o utilizando otras vías de inserción, también se sugiere evitar esta vía cuando se sienta resistencia al intentar introducir un catéter por punción subclavia. ${ }^{10}$

La complicación no infecciosa reportada con mayor frecuencia es la oclusión del catéter, se presenta entre 14 y $36 \%$ de los catéteres, ${ }^{15}$ puede ser parcial -no es posible obtener retorno pero sí infundir soluciones- o total -no hay retorno ni paso de solución a través del catéter-. En nuestra serie encontramos oclusión parcial en ocho pacientes en los cuales fue posible continuar con el uso del catéter hasta terminar el tratamiento, y otra paciente en la cual se suspendió el flujo a través del catéter durante ocho horas sin colocar sello de heparina y sufrió oclusión total del mismo, se intentó terapia con trombolíticos, que no fue exitosa, por lo que se hizo cambio de catéter.

La infección de los puertos es la complicación reportada con mayor frecuencia; ${ }^{16}$ sin embargo, ha sido, por largo tiempo, motivo de controversia, ya que para algunos estudios engloba, desde una infección superficial localizada, hasta una septicemia y para otros solo se incluyen pacientes con un cuadro clínico caracterizado por fiebre y calosfríos sin evidencia de otro foco infeccioso, ${ }^{17}$ por lo que el CDC de Atlanta estandarizó los criterios para definir seis tipos de infecciones asociadas a catéteres centrales:

a) La infección en el sitio de salida del catéter, caracterizada por eritema, hipersensibilidad e inflamación o exudado en el sitio de salida y fiebre, generalmente secundario a cuidado inadecuado del catéter; puede tratarse con antibióticos locales.

b) La infección del reservorio, también relacionada con cuidado inadecuado del 
puerto, se presenta con enrojecimiento, sensibilidad y exudado o celulitis alrededor del reservorio, con o sin la presencia de fiebre, estos pacientes requieren cuidados locales en la piel, antibióticos sistémicos instilados a través del catéter y posible remoción del mismo - como sucedió con nuestro paciente-(figura 5).

c) Infección del túnel, que se presenta sobre el trayecto subcutáneo del catéter a 2.0 $\mathrm{cm}$ del puerto con enrojecimiento, sensibilidad e inflamación o exudado a través del sitio de salida, estos pacientes requerirán de antibióticos sistémicos y remoción del catéter.

d) Colonización del catéter, esto también por técnica deficiente en el manejo del mismo, se identifica mediante cultivos de la punta del catéter, no requiere tratamiento pero puede evitarse con el uso de técnica aséptica cuidadosa al manipular el puerto.

e) Bacteremia asociada al catéter, por técnica deficiente en la colocación de las líneas o por el uso de soluciones contaminadas, se presenta con dolor, enrojecimiento o exudado en el sitio de salida, así como fiebre o escalofrío, se aísla el mismo germen en el cultivo del catéter y el hemocultivo, estos pacientes requieren de manejo sistémico con antibióticos instilados a través del catéter y remoción del mismo.

f) Bacteremia causada por nutrición parenteral contaminada, deberá aislarse el mismo germen en hemocultivo y en la solución, el tratamiento consiste en remoción del catéter y antibióticos sistémicos.

Las infecciones en el catéter se reportan en 0,65 por 1000días de permanencia con una frecuencia de retiro del catéter entre 3 y $28 \%$ por esta causa. ${ }^{12,18}$ La mejor práctica para prevenir esta complicación es utilizar una estricta técnica de asepsia. En nuestro grupo la frecuencia de infecciones que obligaron a retirar el puerto fue menor a la reportada en la literatura internacional debido a que los catéteres son solo mani- pulados por equipos fijos - las mismas personas haciendo las mismas actividades- en nuestro caso el germen aislado fue estafilococo epidermidis por lo que el paciente recibió vancomicina por dos semanas.

Una complicación poco reportada es la exteriorización del puerto, la frecuencia de este evento no se conoce en adultos pero en niños se ha reportado en $1.67 \%,{ }^{19}$ este evento consiste en la lesión de la piel sobre el puerto, frecuentemente relacionado con dehiscencia de la herida quirúrgica, punciones muy frecuentes o pérdida de tejido por disminución rápida de peso ${ }^{16}$-como sucedió en nuestro paciente-(figura 6). El puerto debe ser retirado y si el paciente lo requiere se deberá colocar un catéter en una vía alterna. Se ha sugerido que esta complicación puede evitarse si se utilizan puertos de bajo perfil y se deja suficiente tejido subcutáneo por arriba del reservorio, 20 incluso se ha propuesto colocarlos por debajo de la aponeurosis y lejos de la incisión. ${ }^{19}$

La manipulación de un puerto puede causar retracción del catéter al tejido celular subcutáneo (figura 7), una vez en estas condiciones el catéter queda inutilizado y deberá recolocarse, ${ }^{20}$ en nuestra serie encontramos esta complicación en dos catéteres, ambos con pacientes que desarrollaron un síndrome de vena cava superior, por lo que consideramos que pudieron retraerse hacia la vena cefálica y de ahí al tejido subcutáneo por la presión ejercida al pasar soluciones a través de bomba de infusión. Dos pacientes presentaron rotación del puerto subcutáneo, lo que hizo que no pudieran canalizarse a través de punción, por lo que requirieron de recolocación del mismo, la causa probable fue haber fijado el puerto con material de sutura absorbible. Esta complicación ha sido reportada por otros. ${ }^{21}$

En total se tuvieron 10/99 (10.1\%) complicaciones menores y $6 / 99$ (6\%) mayores lo que constituye una frecuencia de $16 \%$, que se encuentra dentro del rango aceptado en la literatura que fluctúa entre 11 y $25 \% .{ }^{21}$ 


\section{Conclusiones}

Nuestra experiencia corrobora que los catéteres con reservorio subcutáneo para uso prolongado son seguros. Preferimos la colocación a través de disección de la vena cefálica en el surco delto-pectoral porque tienen menor frecuencia de complicaciones que pudieran ser letales - migración del catéter al ventrículo que puede ocasionar arritmias o neumotórax-. El manejo cuidadoso por personal entrenado disminuye el riesgo de complicaciones infecciosas.

\section{Bibliografía}

1. Legha SS, Haq $M$, Rabinowits $M$, et al. Evaluation of silicone elastomer catheters for long-term intravenous chemotherapy. Arch Int Med 1985; 145: 1208 - 1211

2. Carlo JT, Lamont JP, McCarty TM, Livingston S, Kuhn JA. A prospective randomized trial demonstrating valved implantable ports have fewer complications and lower overall cost than nonvalved implantable ports. Am J Surg 2004; 188: 722 - 727

3. DiCarlo I, Pulvirenti E, Mannino M, Toro A. Increased use of percutaneous technique for totally implantable venous access devices. Is it real? A 27 -year comprehensive review on early complications. Ann Surg Oncol 2010; 17: 1649 - 1656

4. Torres PJL, Gerson R, Villalobos A, Serrano A, González JM, Garmilla J, Ramírez C. Reservorio venoso subcutáneo. Reporte de 105 casos. An Med (Mex) 2006;51:11-17

5. Calvo $R$, Ruiz-Giménez JF, Robio $V$, Melmonte $M$, Ruz $A$, Lluch $M$. Reservorios subcutáneos venosos centrales permanentes. Complicaciones. Rev Soc Esp Dolor 2004;11: 150 - 155

6. Kusminsnsky RE. Complications of central venous catheterization. J Am Coll Surg 2007; 4: 681 - 689

7. Chang HM, Hsieh CB, Hsieh HF, Chen TW, Chen CJ Chan DC, el al. An alternative technique for totally implantable central venous access devices. A retrospective study of 1311 cases. Eur J Surg Oncol2006; 32:90 - 93

8. Jablon LK, Ugolini KR, Nahmias NC. Cephalic vein cut-down verses percutaneous access: a retrospective study of complications of implantable venous access devices. Am J Surg 2006; 192:63-67

9. Knebel P, Lopez-Benitez $R$, Fischer $L$, Radeleff BA, Stampfl U, Bruckner T, Hennes R, Kieser M, Kauczor $\mathrm{H}$, Büchler MW, Seiler CM. Insertion of Totally Implantable Venous Access Devices: An Expertise-Based, Randomized, Controlled Trial (NCT00600444) Annals of Surgery 2011: 253; 1111-1117
10. Galloway S, and Bodenham A. Long-term central venous access. Br J Anaesth 2004; 92: 722 - 734

11. Kim DI,Han SH. Venous variations in neck region: Cephalic Vein. International Journal of Anatomical Variations (2010) 3: 208-210

12. Rojas AG, Gerson R, Cervantes J, Flores C, Villalobos A. Acceso vascular en el paciente oncológico. Experiencia de 200 casos. Cir Ciruj 1999; 67: 200 - 204

13. Robles VC, Enriquez E, Cobos E, Guzman NR, Segui $\mathrm{VP}$, Olivares $\mathrm{MH}$. Emboliismo iatrogénico de un catéter a permanencia. AnnMed(Mex)2005;50:35 - 38

14. Andris D, Krzywda E, Schulte W, Ausman R, Quebbeman E.Pinch-off syndrome: a rare etiology for central venous catheter occlusion. J Parenter Enteral Nutr 1999; 18: $531 \pm 3$

15. Baskin JL, Reiss U, Williams JA, Metzger ML, Ribeiro RC, Pui C-H, Howard SC. Thromboliitic therapy for central venous catheter occlusion.DOI: 10.3324/ haematol.2011.050492

16. Vasques $\mathrm{Cl}$, Diniz dos Reis $\mathrm{PE}$, Campos deCarvalho E. Management of totally implanted catheter in patients with cancer: an integrative review. Acta Paul Enferm 2009;22:696-701.

17. Kehr J, Castillo L, Lafourcade RM. Complicaciones infecciosas asociadas a catéter venoso central. RevChilena de Cirugía 2002;54:216 - 224

18. McNelis J, Zarcone J, Marini C, Jurkiewicz A. Outcome of subcutaneously implanted catheters in a teaching hospital. Am J Med Qual 2002; 17:185-8.

19. Bass J, Halton JM. Skin erosion over totally-implanted vascular access-devices in children. Sem Ped Surg $18 ; 84-86$

20. Shulmeister L. Management of non-infectious central venous access device complications.Semm Oncol Nurs2010; 26: 132 - 141

21. Rouzrokh M, Shamsian BS, Tabari AKN, Mahmood M, Kouranlo J, Manafzadeh G, Arzanian MT, Fallah F, Anoush $M$, Gorji FA. Totally implantable subpectoral vs subcutaneous port systems in children with malignant diseases. Arch Iranian Med2009; 12: 389 - 394 


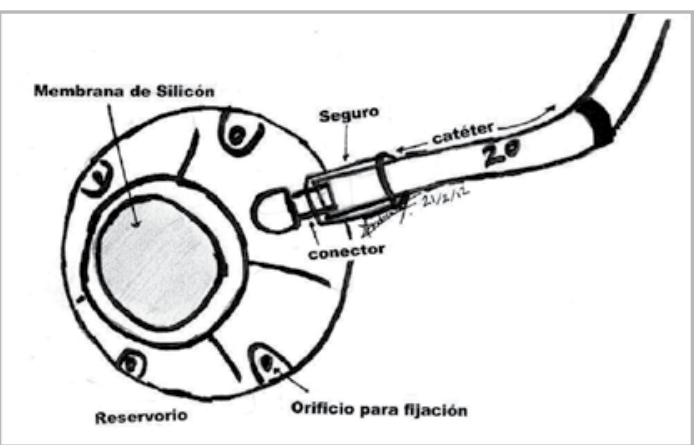

Figura 1. Esquema

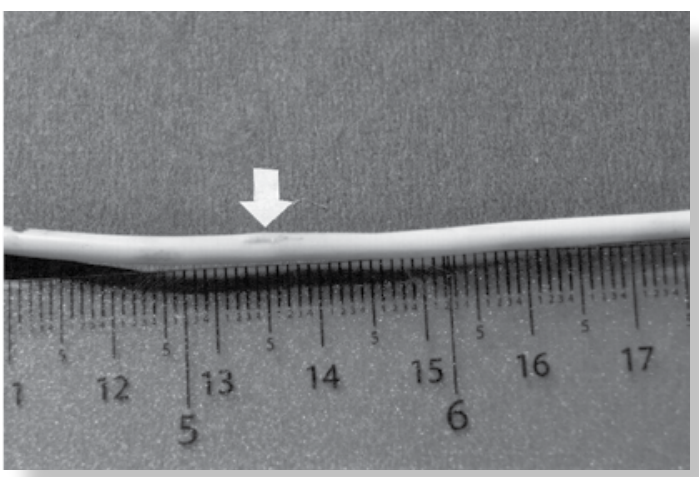

Figura 2

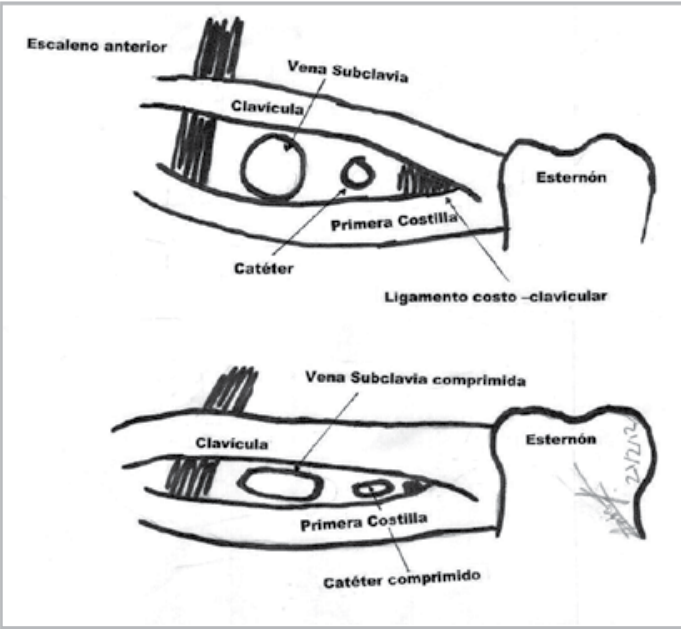

Figura 3. Esquema que muestra

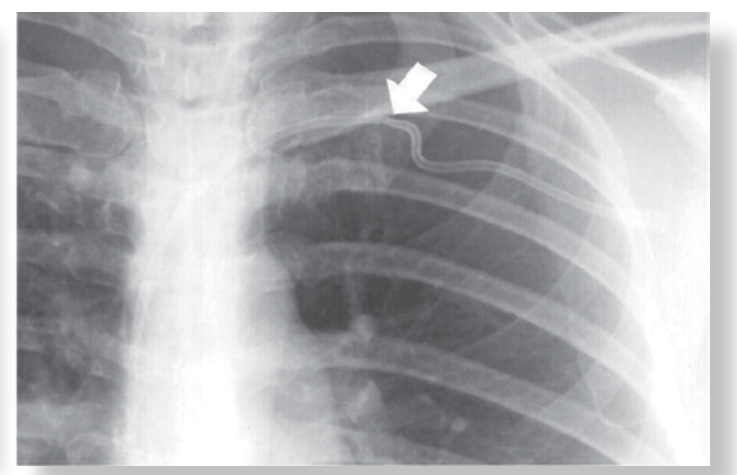

Figura 4

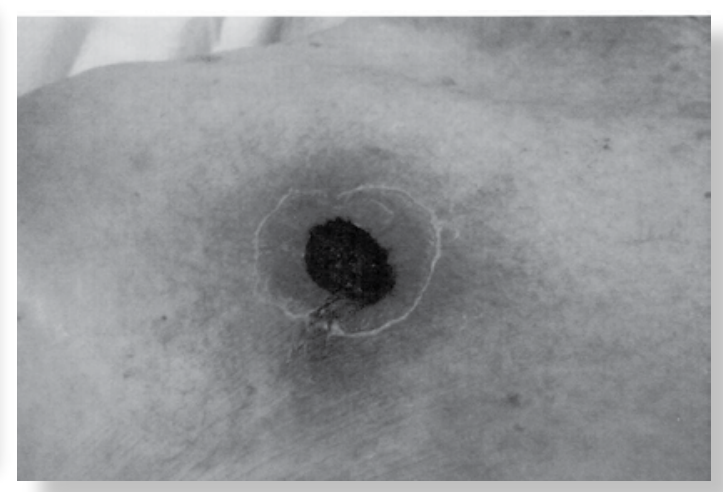

Figura 5

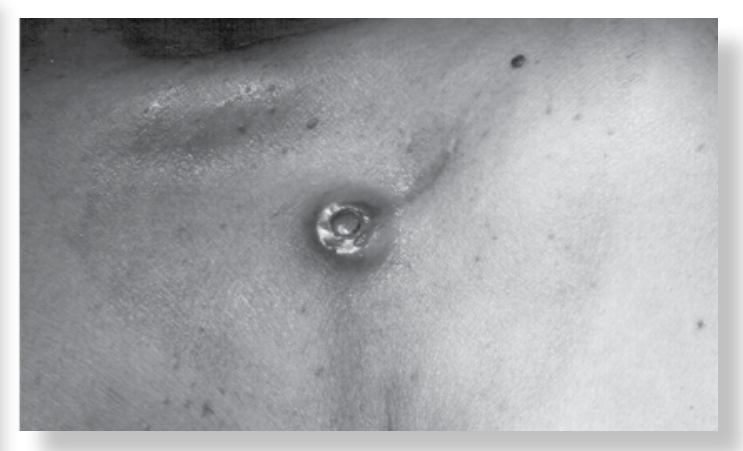

Figura 6

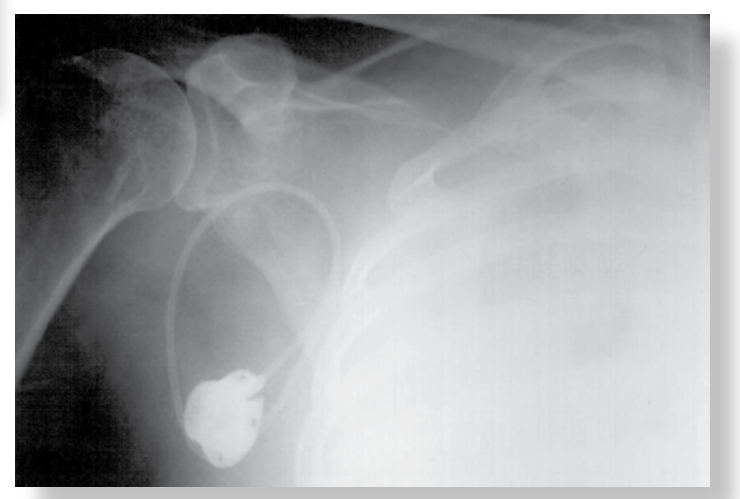

Figura 7 